\title{
Comparación de la corrosividad atmosférica del acero al carbono en
}

\section{Colombia y $\mathrm{Cuba}^{(\cdot)}$}

\author{
Juan Delgado Lastra*, Julio César Minotas Ruiz*, Carlos Enrique Arroyave Posada*, \\ Antonio Mendoza Gómez** y Francisco Corvo Pérez**
}

\begin{abstract}
Resumen Se expusieron a la atmósfera probetas de aceros estructurales de bajo contenido de carbono, en varios sitios de Colombia y Cuba, que se retiraron a intervalos de 2, 3, 6, 12, 18 y 24 meses. Se determinó la corrosión por pérdida de masa y se buscaron relaciones de causalidad con el tiempo de exposición y las cantidades de cloruro y óxidos de azufre depositados. El análisis permitió ajustar modelos de regresión múltiple y también, a través de variables dicótomas, realizar la comparación de la corrosión atmosférica entre Colombia y Cuba, mediante análisis de covarianza.
\end{abstract}

Palabras clave Corrosión atmosférica. Acero al carbono. Métodos estadísticos aplicados a la corrosión. Efecto de los contaminantes sobre la corrosión. Efectos climáticos sobre la corrosión atmosférica.

\section{Comparison of corrosivity of plain carbon steel in Colombia and Cuba}

\begin{abstract}
Samples of structural carbon steel were exposed to the atmosphere in a number of sites in Colombia and Cuba for periods of 2, 3, 6, 12 and 24 months. Corrosion rate was determined by mass loss measurements, and cause - effect relationship to exposure time and pollutants (deposited chloride and sulfur oxides) were calculated. Analysis let to adjust models by the multiple regression method. Also, comparison of atmospheric corrosion rates between Colombia and Cuba were done using covariance analysis.

Keywords

Atmospheric corrosion Carbon steel. Statistical methods applied to corrosion. Contaminant effect on corrosion. Climate effects on atmospheric corrosion.
\end{abstract}

\section{INTRODUCCIÓN}

La búsqueda de un desarrollo verdaderamente sostenible, con métodos de producción más limpios, pasa por la necesidad de lograr que los materiales en los que el hombre soporta su bienestar, presenten una buena estabilidad, de manera que se garanticen largos años de utilización, reduciendo el impacto de la corrosión sobre el ambiente y, a la vez, minimizando la necesidad de consumir nuevas materias primas y recursos energéticos para la producción de los materiales de reemplazo, por lo general mediante procesos altamente contaminantes. Obligatoriamente, esta situación se liga con aspectos económicos y es por lo que se habla de los altos costos que genera la corrosión ${ }^{[1]}$, de los cuales los debidos al deterioro de los materiales en la atmósfera, constituyen un porcentaje significativo ${ }^{[2]}$.

Lo anterior ha sido razón suficiente para que, desde hace muchos años, los estudios de corrosión atmosférica hayan tratado de responder a una pregunta, quizás la más práctica, relacionada con la posibilidad de predecir la duración que tendrán los materiales al ser usados en determinado sitio. Para ello, se ha recurrido al empleo de diversas metodologías, entre las que resaltan el levantamiento de mapas de corrosividad a partir de datos meteorológicos $^{[3]}$, y las mediciones directas de corrosión en sitios representativos ${ }^{[4-12]}$.

(•) Trabajo recibido el día 29 de septiembre de 2000 y aceptado en su forma final el día 17 de abril de 2001.

(*) Grupo de Corrosión y Protección, Universidad de Antioquia, AA 1226, Medellín-Colombia

(**) Centro Nacional de Investigaciones Científicas, Av. 25 y calle 158, La Habana-Cuba 
Mucho más antigua es la idea de establecer modelos matemáticos que permitan calcular la cinética del deterioro, de forma simple y precisa. Resultado del trabajo de varias décadas alrededor del tema, es la ecuación sustentada por investigaciones como las de Hayne y Upham ${ }^{[13]}$ y Pourbaix ${ }^{[14]}$, según la cual la corrosión de un metal, después de varios años de exposición en un sitio dado, es función del tiempo:

$$
\mathrm{C}=a t^{b}
$$

Ecuación que puede rescribirse como:

$$
\ln C=a+b \ln t
$$

por lo que se conoce como la ecuación bilogarítmica de la corrosión atmosférica, donde $a$ corresponde a la corrosión en el primer año (sería la medida de la reactividad inicial), $b$ muestra el efecto del tiempo de exposición (el cambio en el impacto de la atmósfera con el tiempo)' y ambas son funciones representativas de un sitio dado, que parecen depender del tipo de material ${ }^{[15]}$, de la naturaleza de los productos de corrosión que se forman ${ }^{[16]}$, y de los factores medioambientales ${ }^{[17]}$. Con estas consideraciones, se han planteado ecuaciones que, sin embargo, no explican de forma convincente las posibles relaciones y dejan mucho que desear, principalmente en el caso del coeficiente $b$, tal como lo han demostrado Feliu et al. ${ }^{[18 \text { y } 19]}$.

En consecuencia, al recopilar ecuaciones establecidas por diversos autores, se ha encontrado una gran variedad, con diferencias difíciles de susten$\operatorname{tar}^{[2}$ y 9$]$. Esto obliga a pensar que las variables que se han considerado tradicionalmente, no son las más adecuadas; que hay parámetros sin identificar, que afectan de manera importante al coeficiente $b$ y que es necesario encontrar modelos estadísticos más acordes con la situación que se presenta al tener los materiales en la atmósfera, que consideren, por ejemplo, los efectos combinados. Dichas dudas se acentúan al considerar el mecanismo de corrosión atmosférica del acero, propuesto por Stratmann et al. ${ }^{[20-23]}$, según el cual, aunque para que se dé corrosión es necesario que haya humectación, el paso determinante en la cantidad de material corroído es el del secado, razón por la que podrían ser mucho más importantes las características de los ciclos de humectación y secado, que los valores de la humedad relativa o del tiempo de humectación. Además, se debe recordar que el verdadero valor de éste depende grandemente de otros factores co- mo la naturaleza de la superficie del material, el tipo de productos de corrosión, la temperatura, la radiación y la naturaleza y concentración de los contaminantes; situación que ha conducido a buscar alternativas de manejo de los datos de humectación que se vienen utilizando con base en las normas establecidas ${ }^{[24-27]}$.

Curiosamente, varias de las ecuaciones más recientemente desarrolladas para el acero en atmósferas con algún grado de contaminación por óxidos de azufre o por cloruros no incluyen la humedad como factor determinante ${ }^{[9]}$. Esto lleva a suponer que, en dichas situaciones, superado el umbral de la humedad relativa crítica, la incidencia del espesor de la película de electrólito es secundaria frente a la que tienen los contaminantes. Ejemplo de esta situación es el resultado de un vasto estudio efectuado en la Península Ibérica y varios países de América, en el que se hicieron ajustes de datos provenientes de 75 estaciones, que permitieron concluir que las variables más significativas en la ecuación dosis/respuesta o función de daño del acero al carbono eran las velocidades de depósito de $\mathrm{SO}_{2}$ y $\mathrm{Cl}$. En otras palabras, se podía afirmar que la participación de los factores meteorológicos era secundaria, o que la corrosión atmosférica es mucho más sensible a la contaminación de lo que se consideraba previamente. En consecuencia, se estableció la ecuación ${ }^{[9]}$ :

$$
\mathrm{C}=2,49 \cdot 1,59 \mathrm{~S}+0,96 \mathrm{Cl}
$$

donde $\mathrm{C}$ es la velocidad de corrosión del acero, expresada en $\mu \mathrm{m} a^{-1}$, y $\mathrm{S}$ y $\mathrm{Cl}$ corresponden a las tasas de depósito de $\mathrm{SO}_{2}$ y $\mathrm{Cl}$, en $\mathrm{mg} \mathrm{m}^{-2} \mathrm{~d}^{-1}$, respectivamente.

Entre los ejemplos confirmatorios de lo anterior se pueden citar la ecuación obtenida para Galicia, en España ${ }^{[6]}$, y la encontrada para la península de Yucatán, en México ${ }^{[28]}$, caso éste en el que los investigadores concluyeron que, en las condiciones de temperatura y humedad que se presentan en el Caribe mexicano, el $\mathrm{SO}_{2}$ es el factor de mayor influencia en la corrosión del acero.

En general, los coeficientes de correlación múltiple de las ecuaciones que se han estado deduciendo se encuentran en el rango de 0,6 a 0,8, cifras que llaman la atención sobre la necesidad de continuar profundizando en la interpretación de los fenómenos de corrosión atmosférica con miras al establecimiento de ecuaciones cada vez más útiles en la predicción del comportamiento de los materiales en servicio. 
Ante esta panorámica, se ha considerado importante fortalecer los estudios alrededor de una región como la del mar Caribe en la que, características climáticas sui géneris debidas a factores como los altos valores de temperatura, humedad, radiación, vientos, etc., a lo largo de todo el año, son responsables de velocidades de corrosión atmosférica habitualmente preocupantes. En consecuencia, además de los ya mencionados, se han publicado, recientemente, varios trabajos que apuntan a enriquecer el conocimiento existente ${ }^{[29-31]}$. En este mismo sentido se ha orientado el trabajo que aquí se presenta y que está centrado en la comparación del comportamiento de los aceros al carbono en varias estaciones marinas de Colombia y Cuba, mediante modelos de regresión, nunca aplicados, anteriormente, en este tipo de estudios en los que, básicamente, se consideraron los parámetros que se han mostrado de mayor incidencia en la región: tiempo y cantidad de contaminantes depositados. Para ello, se partió de una función dosis-respuesta deducible de la ecuación bilogarítmica, más general que las usualmente presentadas en la literatura, del tipo:

$$
\mathrm{C}=\beta_{1} X_{2}^{\beta_{2}} X_{3}^{\beta_{3}} \ldots X_{k}^{\beta_{k}} e^{u}
$$

o sea:

$\ln C=\alpha+\beta_{2} \ln X_{2}+\beta_{3} \ln X_{3} \ldots+\beta_{k} \ln X_{k}+u$

donde:

$\alpha=\ln \beta_{1}$ representa la interceptación,

$\beta_{2} \ldots \beta_{k}$, son los coeficientes de regresión parciales, $X_{2}, X_{3} \ldots X_{k}$, son los factores explicativos,

$u$, es el término de perturbación estocástica.

\section{CONSIDERACIONES ACERCA DE LA REGRE- SIÓN CON VARIABLES DICÓTOMAS}

En los análisis de regresión, a veces, es necesario tener en cuenta variables explicativas que, por su naturaleza, son cualitativas. Entre ellas, en el caso de la corrosión atmosférica, se consideran la clase de material, el tipo de corrosión, la estación de muestreo, etc. Usualmente, estas variables indican la presencia o ausencia de una cualidad o atributo (v.g. material corroído o no corroído). Para cuantificar estos atributos, se pueden construir variables artificiales que puedan tomar valores 0 o 1 , el primero indicando ausencia y el otro presencia del atributo. Por ejemplo, el 0 puede indicar si la co- rrosión es generalizada y el 1 si es localizada. El 0 puede indicar si la estación de muestreo es costera y el 1 si es del interior, etc. Las variables que adquieren estos valores se llaman variables dicótomas, indicadoras, categóricas o cualitativas ${ }^{[32]}$. Esto permite plantear ecuaciones de la forma:

$$
y_{i}=\alpha+\beta D_{i}+U_{i}
$$

donde:

$y_{\mathrm{i}: \text { pérdida de masa }}$

$D_{i}\left\{\begin{array}{l}1, \text { si la probeta fue observada en estación } \\ \text { costera } \\ 0, \text { si la probeta fue observada en estación } \\ \text { del interior }\end{array}\right.$

$U_{i}$ : término de perturbación estocástica

De la ecuación (6) se deriva que $E\left[y_{i} / D_{i}=0\right]=\alpha$, $E\left[y_{i} / D_{i}^{\prime}=1\right]=\alpha+\beta$, donde $E\left[y_{i} / D_{i}\right]$ es la esperanza matemática condicional de la variable aleatoria $y_{i}$, dado $D_{i}$. En consecuencia, $\alpha$ puede corresponder a la pérdida de masa promedio del material en la estación del interior y $\beta$ representar en cuánto difiere la pérdida de masa promedio en la costa, de la del interior. Así, el promedio de pérdida de masa en la costa estaría representado por $\alpha+\beta$.

Una prueba de hipótesis de que no hay diferencia en la pérdida de masa de los materiales debido a que la estación sea costera o del interior, sería $H_{0}: \beta=0$, a la cual se le puede dar respuesta con base en la prueba $t$.

Supóngase ahora que:

$$
y_{i}=\alpha_{1}+\alpha_{2} D_{i}+\beta X_{i}+u_{i}
$$

donde $X_{i}$ es el tiempo de exposición.

Entonces,

$E\left[y_{i} / X_{i}, D_{i}=0\right]=\alpha_{1}+\beta_{1} X_{i}$, promedio de la pérdida de masa en la estación del interior.

$E\left[y_{i} / X_{i}, D_{i}=1\right]=\left(\alpha_{1}+\alpha_{2}\right)+\beta X_{i}$, promedio de la pérdida de masa en la estación de la costa.

La figura 1 ilustra el comportamiento de la pérdida en los dos tipos de estaciones, suponiendo que las pendientes sean iguales. Esto significa que la pérdida de masa promedio difiere de una situación a otra, pero la tasa de crecimiento en el tiempo es la misma.

Si se supone que el modelo de regresión contiene una variable cualitativa con más de dos clases, entonces 


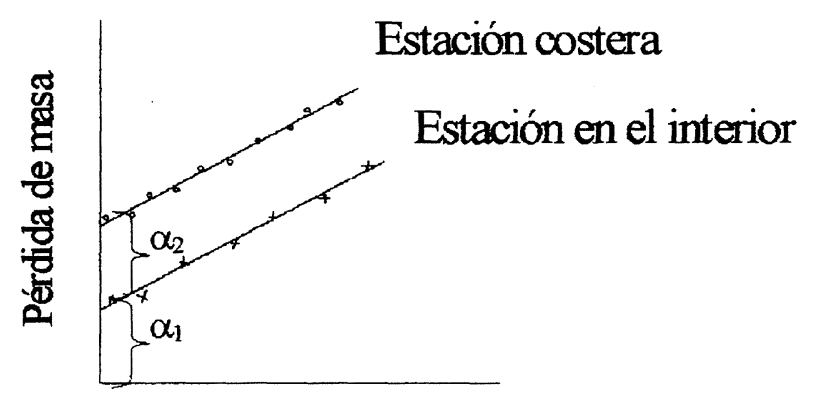

Tiempo

Figura 1. Comparación de la variable respuesta con pendientes iguales.

Figure 1. Comparison of variable-response with equal stopes.

$D_{2 \mathrm{i}}\left\{\begin{array}{c}1 \text { Si es el material } \mathrm{B} \\ 0 \text { Si es otro tipo } \\ \text { de material }\end{array} \quad \mathrm{D}_{3 \mathrm{i}}\left\{\begin{array}{c}1 \text { Si es el material A } \\ 0 \text { Si es otro tipo } \\ \text { de material }\end{array}\right.\right.$

En consecuencia,

$$
y_{i}=\alpha_{1}+\alpha_{2} D_{2 i}+\alpha_{3} D_{3 i}+\beta X i+U i
$$

En este caso,

$$
\begin{aligned}
& E\left[y_{i} / D_{i}=0, D_{3 i}=0, X_{i}\right]=\alpha_{1}+\beta X_{i} \\
& E\left[y_{i} / D_{2 i}=1, D_{3 i}=0, X_{i}\right]=\left(\alpha_{1}+\alpha_{2}\right)+\beta X_{i} \\
& E\left[y_{i} / D_{2 i}=0, D_{3 i}=1, X_{i}\right]=\left(\alpha_{1}+\alpha_{2}\right)+\beta X_{i}
\end{aligned}
$$

La figura 2 muestra el comportamiento de la pérdida de masa para tres materiales diferentes en una misma estación, suponiendo que la tasa de crecimiento en el tiempo es igual.

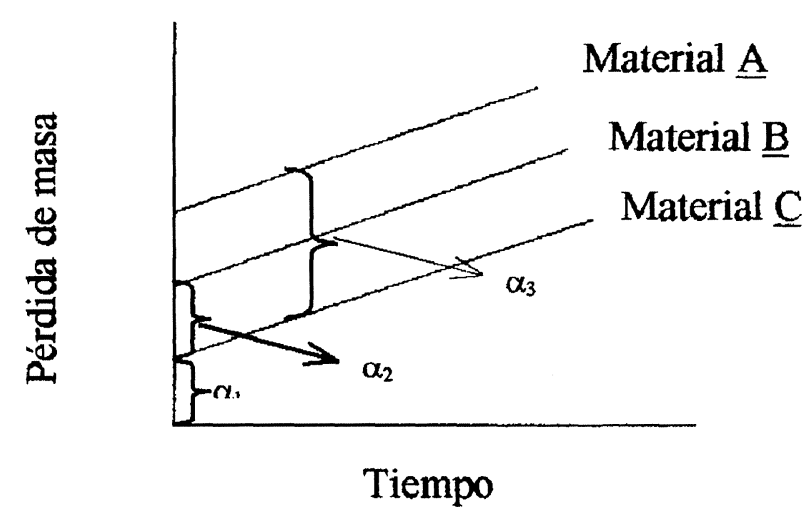

Figura 2. Comparación de la variable respuesta con pendientes iguales para tres categorías.

Figure 2. Comparison of variable-response with equal slopes, in the three categories.
Debe notarse que el número de variables dicótomas para cada variable cualitativa es el número de categorías menos uno.

Supóngase el modelo con una variable cuantitativa y dos cualitativas, como variables explicativas:

$$
y_{i}=\alpha_{1}+\alpha_{2} D_{i}+\alpha_{3} D_{3 i}+\beta X_{i}+U_{i}
$$

Donde:

$D_{2 \mathrm{i}}\left\{\begin{array}{l}1 \text { para el material } A \\ 0 \text { para el material B }\end{array} D_{3 \mathrm{i}}\left\{\begin{array}{c}1 \text { si la estación es } \\ \text { costera } \\ 0 \text { si la estación es } \\ \text { del interior }\end{array}\right.\right.$

Los promedios de pérdidas de masa serán:

Material $\mathrm{B}$ en el interior:

$E\left[y / D_{2}=0, D_{2}=0, X_{i}\right]=\alpha_{1}+\beta X_{i}$

Material $\mathrm{A}$ en el interior:

$E\left[y / D_{2}=1, D_{3}=0, X_{i}\right]=\left(\alpha_{1}+\alpha_{2}\right)+\beta X_{i}$

Material $B$ en la costa:

$E\left[y / D_{2}=0, D_{3}=1, X_{i}\right]=\left(\alpha_{1}+\alpha_{3}\right)+\beta X_{i}$

Material A en la costa:

$E\left[y / D_{2}=1, D_{3}=1, X_{i}\right]=\left(\alpha_{1}+\alpha_{2}+\alpha_{3}\right)+\beta_{i} X_{i}$

En los casos anteriores se supuso que la pendiente $\beta$ era la misma para cada una de las categorías de las variables cualitativas en el respectivo modelo de regresión; sin embargo, resulta de gran importancia probar si el coeficiente diferencial de pendiente es significativo o no y, así, poder realizar la comparación de dos regresiones. En efecto, supóngase:

$$
y_{i}=\alpha_{1}+\alpha_{2} D_{i}+\beta_{1} X_{i}+\beta_{2}\left(D_{i} X_{i}\right)+U_{i}
$$

Donde:

$D_{i}\left\{\begin{array}{l}1, \text { si el material es } \mathrm{A} \text { o la estación es costera } \\ 0, \text { si el material es } \mathrm{B} \text { o la estación es interior }\end{array}\right.$

Al reunir todas las observaciones, bien sea de los dos tipos de materiales o de las dos estaciones, se tiene:

$$
\begin{aligned}
& E\left[y_{i} / D_{i}=0, X_{i}\right]=\alpha_{1}+\beta_{1} X_{i} \\
& E\left[y_{i} / D_{i}=1, X_{i}\right]=\left(\alpha_{1}+\alpha_{2}\right)+\left(\beta_{1}+\beta_{2}\right) X_{i}
\end{aligned}
$$

Donde $\alpha_{2}$ es la interceptación diferencial y $\beta_{2}$ el coeficiente diferencial de pendiente, que indican en cuánto difieren la intercepción y la pendiente, respectivamente. 
La comparación de regresiones también admite contar con varias variables explicativas cuantitativas y con varias cualitativas con dos o más categorías; esto es, un modelo de regresión lineal múltiple, el cual se puede ajustar por mínimos cuadrados ordinarios $(\mathrm{MCO})$ y es representable por la ecuación:

$$
\begin{gathered}
y_{i}=\beta_{1}+\beta_{2} X_{2 i}+\beta_{3} X_{3 i}+\ldots+\beta_{k} X_{k i}+U_{i} \\
i=1,2,3 \ldots, n
\end{gathered}
$$

La estimación de este modelo por MCO permite los siguientes supuestos:

- $E\left[U_{i}\right]=0$, o sea, que el valor medio de la perturbación $U_{i}$ es igual a cero

- $\operatorname{Var}\left(U_{i}\right)=E\left[U_{i}^{2}\right]=\sigma^{2}$, o sea que hay homoscedasticidad o igual varianza de los $U i$.

- $\operatorname{Cov}\left(U_{i} U_{j}\right)=0$, con $i \neq j$, o sea que no hay autocorrelación entre las perturbaciones.

- $X_{2}, X_{3}, \ldots, X_{k}$ son fijas o no estocásticas.

- No hay relación lineal exacta entre las variables $X$, es decir, no hay multicolinealidad.

- El modelo de regresión debe estar correctamente especificado.

El modelo clásico de regresión lineal múltiple supone, además, que los $U_{i}$ se distribuyen $N$ $\left(0, \sigma^{2}\right)$. Esto permite realizar las hipótesis pertinentes para el ajuste del modelo.

\section{APLICACIÓN DEL MODELO A LOS DATOS DE COLOMBIA Y CUBA}

En el trabajo que aquí se presenta, se expusieron probetas de acero estructural de bajo carbono, de $150 \times 100 \mathrm{~mm}$, en las estaciones colombianas de Mamonal (E-1), Base Naval (E-2) e Isla Naval (E3) y las cubanas de Viriato (E-4), Holguín 1 (E-5), Holguín 2 (E-6) y Holguín 3 (E-7), tal como se señala en la figura 3.

Periódicamente $(3,6,12,18$ y 24 meses, y en algunos casos un mes), se evaluaron la tasa de corrosión, expresada como pérdida de masa (en mg) y el depósito de sulfatos $\left(\mathrm{SO}_{2}\right.$, en $\left.\mathrm{mg} \cdot \mathrm{m}^{-2}\right)$ y cloruros $\left(\mathrm{Cl}\right.$, en $\left.\mathrm{mg} \cdot \mathrm{m}^{-2}\right)$, siguiendo los métodos sugeridos por la norma ISO TC 156. La tabla I presenta las características generales de cada una de las estaciones.

Siguiendo la norma citada, en cada período se retiraron tres réplicas, a las que se evaluó la pérdida de masa. Estos resultados, junto con los de depósito de contaminantes para los mismos tiempos

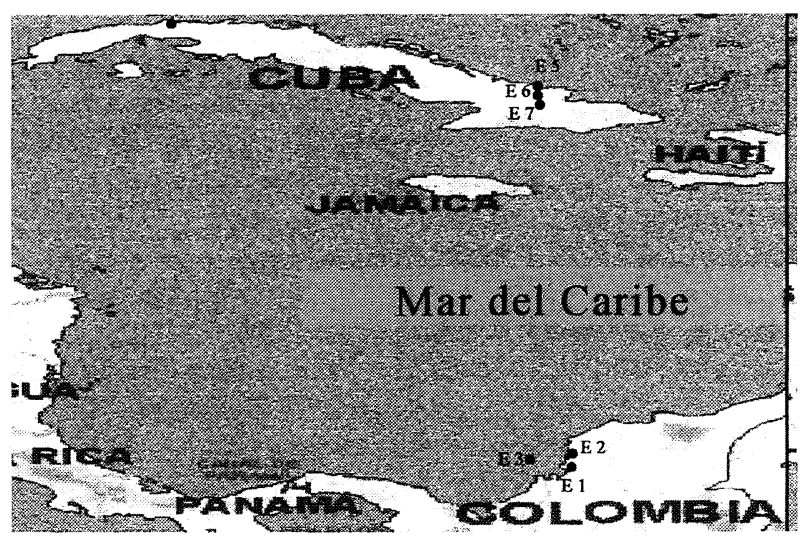

Figura 3. Ubicación geográfica de las estaciones analizadas en el estudio.

Figure 3. Position of the stations used in this study.

Tabla I. Estaciones consideradas en el estudio

Table I. Identification of the stations used in the study

\begin{tabular}{ll}
\hline Estación & Características \\
\hline 1. Mamonal & Marina industrial \\
2. Base naval & Marina urbana \\
3. Isla naval & Marina rural \\
4.Viriato & Marina urbana, con salpicadura \\
5. Holguín 1 & Marina rural $50 \mathrm{~m}$ del litoral \\
6. Holguín 2 & Marina rural $500 \mathrm{~m}$ del litoral \\
7. Holguín 3 & Marina rural $1.132 \mathrm{~m}$ del litoral \\
\hline
\end{tabular}

de exposición, están reflejados en la tabla II. Esto último significa que se fueron acumulando los datos de depósito de contaminantes que se midieron mensualmente, con el fin de hacerlos coherentes con la acumulación de tiempos y de deterioro del acero. Por otro lado, para no perder la variabilidad de las réplicas en la variable respuesta, se trabajó con valores repetidos en las variables explicativas correspondientes.

De acuerdo con las consideraciones presentadas, el modelo que se ajusta a este experimento es de la forma:

$$
y_{i}=\beta_{1} X_{2 i}^{\beta_{2}} X_{3 i}^{\beta_{3}} \ldots X_{k i}^{\beta k} e^{u_{i}}
$$

Ecuación que se linealiza mediante la transformación logarítmica, como:

$$
\operatorname{Ln} y_{i}=\alpha+\beta_{2} \operatorname{Ln} X_{2 i}+\beta_{3} \operatorname{Ln} X_{3 i}+\ldots \ldots \ldots \beta_{K} \operatorname{Ln} X_{k i}+u_{i}
$$

donde: $\quad \alpha=\operatorname{Ln} \beta_{1}$ 
Tabla II. Corrosión y contaminantes de las diferentes estaciones

Table II. Corrosion and contaminants of the different stations

\begin{tabular}{|c|c|c|c|c|c|c|c|c|c|}
\hline Estación & $\begin{array}{l}\text { Tiempo } \\
\text { (d) }\end{array}$ & $\begin{array}{c}\text { SOx } \\
\left(\mathrm{mg} \mathrm{m}^{-2}\right)\end{array}$ & $\begin{array}{c}\mathrm{Cl} \\
\left(\mathrm{mg} \mathrm{m}^{-2}\right)\end{array}$ & $\begin{array}{l}\text { Pérdida de masa } \\
\text { acumulada (mg) }\end{array}$ & Estación & $\begin{array}{l}\text { Tiempo } \\
\text { (d) }\end{array}$ & $\begin{array}{c}\text { SOx } \\
\left(\mathrm{mg} \mathrm{m}^{-2}\right)\end{array}$ & $\begin{array}{c}\mathrm{Cl} \\
\left(\mathrm{mg} \mathrm{m}^{-2}\right)\end{array}$ & $\begin{array}{l}\text { Pérdida de masa } \\
\text { acumulada (mg) }\end{array}$ \\
\hline \multirow[t]{12}{*}{ Mamonal } & \multirow{4}{*}{111} & \multirow{4}{*}{$1.147,2$} & \multirow{4}{*}{$4.806,3$} & 867,27 & & \multirow[t]{3}{*}{30} & \multirow[t]{3}{*}{306} & \multirow[t]{3}{*}{762} & 168,04 \\
\hline & & & & 966,44 & & & & & 183,86 \\
\hline & & & & 846,55 & & & & & 179,43 \\
\hline & & & & $1.808,01$ & & \multirow[t]{3}{*}{92} & \multirow[t]{3}{*}{$3.247,6$} & \multirow[t]{3}{*}{$55.853,2$} & 224,37 \\
\hline & \multirow{2}{*}{202} & \multirow{2}{*}{$4.440,7$} & \multirow{2}{*}{$7.898,4$} & $1.795,39$ & & & & & \multirow{2}{*}{$\begin{array}{l}217,08 \\
217,72\end{array}$} \\
\hline & & & & $1.786,55$ & & & & & \\
\hline & 384 & $7.156,24$ & 13.283 & $3.125,7$ & & \multirow[t]{2}{*}{194} & $5.998,48$ & $130.827,78$ & $1.937,03$ \\
\hline & & & & $3.264,1$ & & & & & $1.878,8$ \\
\hline & & & & 3280,64 & & 365 & $11.902,65$ & $317.703,3$ & $\begin{array}{l}7.519,38 \\
7.485\end{array}$ \\
\hline & 741 & $11.764,9$ & $30.988,7$ & $6.593,54$ & & 559 & $13.846,43$ & $457.904,85$ & $9.175,64$ \\
\hline & & & & $6.603,98$ & & & & & \\
\hline & & & & $6.335,65$ & Holguín 1 & & & & \\
\hline Base Naval & & & & & & 30 & 648 & 2.475 & 162,6 \\
\hline & 102 & $1.331,1$ & $5.045,1$ & 349,64 & & & & & 114,6 \\
\hline & & & & 451,38 & & & & & 151 \\
\hline & & & & 283,68 & & 90 & 1.944 & 7.425 & 695,5 \\
\hline & 193 & $2.285,1$ & $11.260,2$ & 718,37 & & & & & 807,1 \\
\hline & & & & 751,56 & & & & & 663,9 \\
\hline & & & & 948,13 & & 180 & $2.901,6$ & 13.887 & $1.446,6$ \\
\hline & 374 & $4.067,7$ & $21.627,6$ & $3.391,78$ & & & & & $1.600,8$ \\
\hline & & & & $2.788,87$ & & & & & 1.599 \\
\hline & & & & $2.781,75$ & & 360 & 5.256 & $40.251,6$ & $2.665,6$ \\
\hline & 731 & $12.872,9$ & $49.388,7$ & $2.724,06$ & & & & & $2.786,9$ \\
\hline & & & & $2.954,55$ & & & & & $2.531,9$ \\
\hline & & & & $3.572,02$ & & 360 & 5.256 & $40.251,6$ & $2.810,1$ \\
\hline Isla Naval & & & & & & & & & $3.060,8$ \\
\hline & 98 & 193,2 & $2.830,8$ & 315,37 & Holguín 2 & & & & \\
\hline & & & & 317,64 & & 30 & 153 & 978 & 48,9 \\
\hline & & & & 340,28 & & & & & 49,3 \\
\hline & 189 & 1.293 & $5.546,4$ & 872,36 & & & & & 51 \\
\hline & & & & 979,79 & & 90 & 459 & 2.934 & 135,9 \\
\hline & & & & 672,69 & & & & & 142,5 \\
\hline & 369 & $3.506,7$ & $12.075,9$ & 827,65 & & & & & 137,5 \\
\hline & & & & 830,8 & & 180 & 572,4 & 3.708 & 243,6 \\
\hline & & & & 826,3 & & & & & 208,3 \\
\hline & 728 & 7.278 & $27.770,1$ & $1.667,81$ & & 360 & & & 210 \\
\hline & & & & $1.668,44$ & & 360 & $1.407,6$ & $9.176,4$ & 287 \\
\hline & & & & $1.676,43$ & & & & & 305,9 \\
\hline Viriato & & & & & & & & & 253,9 \\
\hline & 74 & $1.450,4$ & $18.766,4$ & 117,19 & & 720 & 2.232 & 20.160 & 881,4 \\
\hline & & & & 5208 & & & & & 808,7 \\
\hline & & & & 208,33 & & & & & $1.014,3$ \\
\hline & 192 & 50054 & $49.848,96$ & $\begin{array}{l}200,30 \\
32552\end{array}$ & Holguín 3 & & & & \\
\hline & & & & 325,52 & & 30 & 171 & 519 & 26,8 \\
\hline & & & & & & & & & 29,8 \\
\hline & & & & & & & & & 28,9 \\
\hline & & ב, כוסוט & & $1.692,71$ & & 90 & 513 & 1.557 & 104,7 \\
\hline & & & & $1.236,98$ & & & & & 112 \\
\hline & & & & 1.250 & & & & & 108,4 \\
\hline & 30 & 306 & 762 & 187,34 & & 180 & $1.173,6$ & 3.024 & 171,8 \\
\hline & & & & 177,26 & & & & & 195 \\
\hline & & & & 193,16 & & & & & 173,3 \\
\hline & 92 & $3.247,6$ & $55.853,2$ & 262,51 & & 360 & $2.098,8$ & $4.827,6$ & 249,3 \\
\hline & & & & 251,62 & & & & & 259,4 \\
\hline & & & & 253,64 & & & & & 252,7 \\
\hline & 194 & $5.998,48$ & $130.827,78$ & $1.822,79$ & & 720 & 227,4 & 13.572 & 656,8 \\
\hline & & & & $1.711,82$ & & & & & 721,3 \\
\hline
\end{tabular}


Aplicando la ecuación 13 al proceso específico de la pérdida de masa explicada por el efecto del tiempo y los contaminantes $\mathrm{SO}_{2}$ y $\mathrm{Cl}$, en todas las estaciones, se obtiene:

$$
\begin{aligned}
& \operatorname{LnPerd}=\beta_{1}+\beta_{2} \operatorname{Lnt}+\beta_{3} \operatorname{LnCl}+\beta_{4} \mathrm{LnSO}_{2}+ \\
& \beta_{5} \mathrm{LnClLnSO}_{2}+\beta_{6} \mathrm{D}_{8}+\beta_{7} \mathrm{D}_{9}+\beta_{8} \mathrm{D}_{1}+\beta_{9} \mathrm{D}_{2}+\beta_{10} D_{3} \\
& +\beta_{11} D_{4}+\beta_{12} \operatorname{Ln} t^{*} D_{8}+\beta_{13} \operatorname{Ln} t^{*} D_{9}+\beta_{14} \operatorname{Ln} t^{*} D_{1}+ \\
& \beta_{15} \operatorname{Ln} t^{*} D_{2}+\beta_{16} \operatorname{Ln} t^{*} D_{3}+\beta_{17} \operatorname{Ln} t^{*} D_{4}+ \\
& \beta_{18} \mathrm{LnCl} * D_{8}+\beta_{19} \mathrm{LnCl}^{*} D_{9}+\beta_{20} \mathrm{LnCl} * D_{1}+ \\
& \beta_{21} \mathrm{LnCl} * \mathrm{D}_{2}+\beta_{22} \mathrm{LnCl} * \mathrm{D}_{3}+\beta_{23} \mathrm{LnCl}^{*} \mathrm{D}_{4}+ \\
& \beta_{24} \mathrm{LnSO}_{2} * D_{8}+\beta_{25} \mathrm{LnSO}_{2} * D_{9}+\beta_{26} \mathrm{LnSO}_{2} * D_{1}+ \\
& \beta_{27} \mathrm{LnSO}_{2} * D_{2}+\beta_{28} \mathrm{LnSO}_{2} * D_{3}+ \\
& \beta_{29} \mathrm{LnSO}_{2} * D_{4}+\beta_{30} \mathrm{LnClLnSO}_{2} * D_{8}+ \\
& \beta_{31} \mathrm{LnClLnSO}_{2} * D_{9}+\beta_{32} \mathrm{LnClLnSO}_{2} * D_{1}+ \\
& \beta_{33} \mathrm{LnClLnSO}_{2} * \mathrm{D}_{2}+\beta_{34} \mathrm{LnClLnSO}_{2} * \mathrm{D}_{3}+ \\
& \beta_{35} \mathrm{LnClLnSO}_{2} * D_{4} \text {. }
\end{aligned}
$$

Esto significa que las variables pérdida de masa, tiempo, $\mathrm{Cl}$ y $\mathrm{SO}_{2}$, debieron ser transformadas por el logaritmo natural para obtener un mejor ajuste.

En ocasiones es necesario tener en cuenta las correlaciones entre perturbaciones de diferentes ecuaciones dadas en el tiempo, a las cuales se les conoce como correlaciones contemporáneas. Ésta es una forma distinta de autocorrelación y cuando se presenta, es más eficiente estimar todas las ecuaciones conjuntamente, lo cual se puede realizar a través de los modelos SUR (Seemingly Unrelated Regression Equations) $)^{[33]}$, según los cuales el conjunto de ecuaciones se puede representar como:

$$
y=\chi \vec{\beta}+\vec{u}
$$

Para el caso en el que se tienen tres estaciones, la ecuación 15 adquiere la forma:

$$
\left[\begin{array}{l}
\overrightarrow{y_{1}} \\
\overrightarrow{y_{2}} \\
\overrightarrow{y_{3}}
\end{array}\right]=\left[\begin{array}{ccc}
x_{1} & 0 & 0 \\
0 & x_{2} & 0 \\
0 & 0 & x_{3}
\end{array}\right]\left[\begin{array}{l}
\overrightarrow{\beta_{1}} \\
\overrightarrow{\beta_{2}} \\
\overrightarrow{\beta_{3}}
\end{array}\right]+\left[\begin{array}{l}
\overrightarrow{u_{1}} \\
\overrightarrow{u_{2}} \\
\overrightarrow{u_{3}}
\end{array}\right]
$$

$$
(3 \mathrm{~N} \times 1) \quad(3 \mathrm{~N} \times 9) \quad(9 \times 1) \quad(3 \mathrm{~N} \times 1)
$$

siendo $\vec{y}_{i},(i=1,2,3)$ la pérdida de peso del acero en la estación $i$, de magnitud $(\mathrm{N} \times t)$. Así mismo, $x_{i}$ y 0 son matrices de $\mathrm{N} \times 3 ; \vec{\beta}$, es el vector de coeficientes de regresión parcial de $(3 \times 1)$, y $\vec{u}_{i}$ corresponde al término de perturbación estocástica de $(\mathrm{N} \times 1)$ para la estación $i$.

En este caso, la matriz de var-covs de $u$, denotada por $\Phi$, será:
$\Phi=\operatorname{var}-\operatorname{cov}(\vec{u})=E[\vec{u} \vec{u}]=\Sigma \otimes I_{\mathrm{N}}(3 \mathrm{~N} \times 3 \mathrm{~N})$

donde $I_{\mathrm{N}}$ es la matriz identidad de $\mathrm{N} \times \mathrm{N}, \otimes$ representa el producto Kroneker ${ }^{[34]}$, y

$$
\Sigma=\left[\begin{array}{lll}
\sigma_{11} & \sigma_{12} & \sigma_{13} \\
\sigma_{21} & \sigma_{22} & \sigma_{23} \\
\sigma_{31} & \sigma_{32} & \sigma_{33}
\end{array}\right]
$$

debiendo recordar que $\sigma_{i j}, \forall i \neq j$, representa la covarianza entre dos variables cualesquiera y que es la varianza de una variable aleatoria.

Una prueba suficiente para decidir sobre la aplicación de los modelos SUR fue sugerida por Breusch y Pagan ${ }^{[35]}$, según la cual la hipótesis nula para el caso de tres variables es $H_{0}: \sigma_{12}=\sigma_{13}=$ $\sigma_{23}=0$. Entonces, bajo $H_{0}$ cierta:

$$
\Phi=\Sigma \otimes I_{N}=\left[\begin{array}{lll}
\sigma_{11} & 0 & 0 \\
0 & \sigma_{22} & 0 \\
0 & 0 & \sigma_{33}
\end{array}\right]
$$

y el estadístico de prueba tiene una distribución chi-cuadrado con tres grados de libertad.

Este modelo exige los $n_{\mathrm{i}}$ iguales (o número de observaciones por ecuación). Por lo tanto, fue necesario aplicarlo por separado para las estaciones de Colombia y de Cuba. Además, en Cuba debió retirarse la estación Viriato por tener un número de observaciones diferente al resto.

La realización de esta prueba utilizando el paquete estadístico Shazam ${ }^{[36]}$, arrojó el informe de salida que se presenta en la tabla III. En consecuencia, de acuerdo con los valores $P$ de la prueba de Breusch y Pagan, no se puede rechazar $H_{0}: \sigma_{12}$ $=\sigma_{13}=\sigma_{23}=0$. Es decir, que el modelo planteado inicialmente puede ser ajustado utilizando $\mathrm{MCO}$, sin necesidad de emplear modelos SUR.

En la validación de los supuestos del modelo, se detectaron problemas severos de multicolinealidad; por lo tanto, debió emplearse regresión Ridge, la cual considera estimadores con un pequeño sesgo pero sustancialmente más precisos que los estimadores insesgados, tal como se aprecia en la figura $4^{[35]}$.

Estos estimadores se obtienen introduciendo en las ecuaciones normales un sesgo $\mathrm{C}$, esto es:

$$
\begin{aligned}
& \left(\gamma_{x x}+C I\right) \beta^{R}=\gamma_{y x} \\
& \beta^{R}=\left(\gamma_{x x}+C I\right)^{-1} \gamma_{y x}
\end{aligned}
$$

donde $\beta^{\mathrm{R}}$ es el estimador Ridge de los coeficientes de regresión parciales y $\gamma_{\mathrm{xx}}$ y $\gamma_{\mathrm{yx}}$ son las matrices 
Tabla III. Informe de salida del programa Shazam, según la prueba de Breusch y Pagan Table III. Output report from Shazam analysis, following the Brensch and Pagen LM test

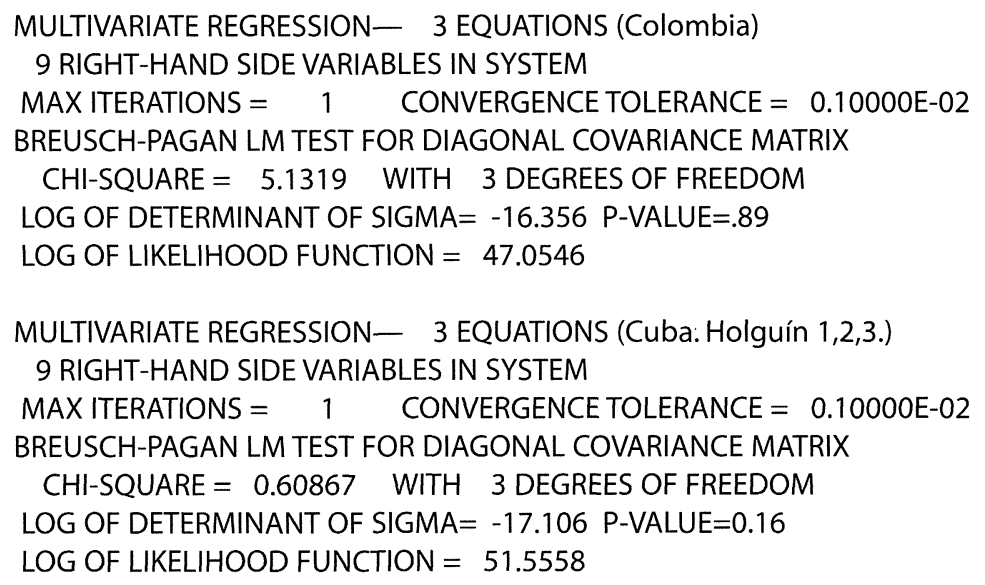

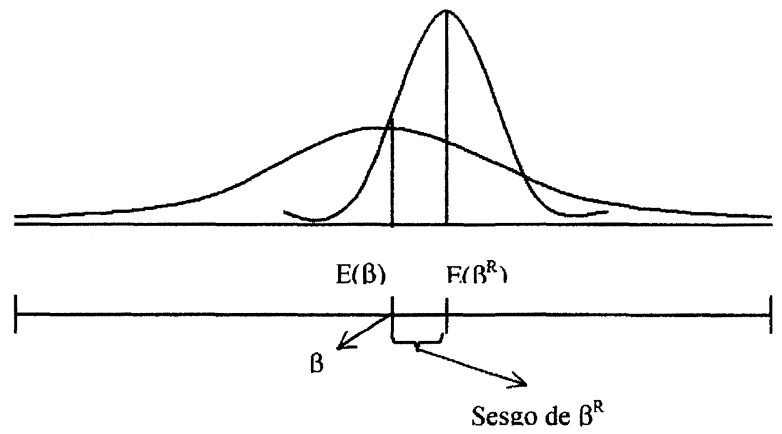

Figura 4. Distribución de los estimadores por regresión Ridge.

Figure 4. Parameter distribution using ridge regression.

X'X y X'Y expresadas en términos de correlaciones.

La constante $\mathrm{C}$ se determina resolviendo el sistema para distintos valores, $0 \leq \mathrm{C} \leq 1$. El valor de $\mathrm{C}$ será aquel donde los estimadores se hacen estables y el factor de inflación de la varianza $\mathrm{VIF}_{\mathrm{k}}=1 /(1$ $\left.\mathrm{R}_{\mathrm{J}}^{2}\right), \mathrm{R}_{\mathrm{J}}^{2}$ : el coeficiente de determinación de $\mathrm{X}_{\mathrm{j}}$ contra el resto de las $\mathrm{X}$, se hace pequeño o cercano a 1 .

\section{RESULTADOS DE LA APLICACIÓN DEL MO- DELO}

Al tomar como base de comparación la estación Holguín 3 y aplicar el análisis por Shazam, se obtuvieron las correlaciones para las diferentes estaciones:
Mamonal:

LnPerd $=0,034829+0,556544 \uparrow \operatorname{Ln} \mathrm{t}+0,119837 \uparrow \mathrm{LnCl}+$

$+0,18424 \uparrow \mathrm{LnSO}_{2}+0,0173124 \uparrow \mathrm{LnCl}^{*} \mathrm{LnSO}_{2}$

Base Naval:

LnPerd $=-0,33238 \uparrow+0,532661 \uparrow \operatorname{Ln} t+0,0997024 \mathrm{LnCl}+$

$+0,1487603 \mathrm{LnSO}_{2}+0,0158226 \uparrow \mathrm{LnCl} * \mathrm{LnSO}_{2}$

Isla Naval:

LnPerd $=-0,2048 \uparrow+0,490788$ Ln $\mathrm{t}+0,089685 \mathrm{LnCl}+$

$+0,130083 \mathrm{LnSO}_{2}+0,012132 \mathrm{LnCl} * \mathrm{LnSO}_{2}$

Viriato:

LnPerd $=0,28737 \uparrow+0,536129 \uparrow$ Ln $\mathrm{t}+0,105 \mathrm{LnCl}+$

$+0,1588786 \mathrm{LnSO}_{2}+0,01167395 \mathrm{LnCl}{ }^{2 n S O} \mathrm{~L}_{2}$

Holguín 1:

LnPerd $=0,2718 \uparrow+0,561064 \uparrow \operatorname{Ln} \mathrm{t}+0,1299 \uparrow \operatorname{LnCl}+$

$+0,18262 \mathrm{LnSO}_{2}+0,0155135 \mathrm{LnCl}^{*} \mathrm{LnSO}_{2}$

Holguín 2:

LnPerd $=0,11854+0,56096$ Ln $\mathrm{t}+0,10881 \mathrm{LnCl}+$ $+0,1544772 \mathrm{LnSO}_{2}+0,012067 \mathrm{LnCl} * \mathrm{LnSO}_{2}$

Holguín 3:

LnPerd $=0,0535+0,50799$ Ln $\mathrm{t}+0,10463 \mathrm{LnCl}+$ $+0,151 \mathrm{LnSO}_{2}+0,012098 \mathrm{LnCl} * \mathrm{LnSO}_{2}$

El símbolo $\uparrow$ señala aquellos coeficientes que tienen diferencias significativas con los de la estación base.

\section{ANÁLISIS DE LOS RESULTADOS}

Después de una exploración exhaustiva, el modelo arrojó un coeficiente de determinación $\mathrm{R}^{2}=0,76$. Éste resultó castigado por el valor seleccionado como sesgo para los estimadores Ridge $(C=0,3)$, donde los coeficientes de inflación de la varianza 
VIFj se acercaban a 1, pero sobre todo, donde las pruebas $t$ individuales mostraban el significado de cada una de las variables involucradas, con coeficientes de regresión parciales, acordes a los principios fisicoquímicos de comportamiento de los materiales en la atmósfera.

Para el conjunto de ecuaciones resultantes, se puede afirmar que si el tiempo, los cloruros y los óxidos de azufre se fijan en 0 , la pérdida de masa promedio es $e^{\hat{\alpha}}$ (de la interceptación), donde se refleja en parte la influencia de todas las variables omitidas en el modelo. No sobra aclarar que el estimador de la interceptación del modelo original es $\hat{\beta}_{1}=$ antilog $\hat{\alpha}$, que es un estimador sesgado.

En la ecuación de la estación base Holguín 3, debe notarse que su interceptación tiene poco aporte y no presenta significado. Sin embargo, su presencia es necesaria para la validación de los supuestos, tal como ocurre para el caso del análisis de autocorrelación.

De los conceptos de elasticidad que brinda la teoría de la transformación logarítmica ${ }^{[32]}$, se puede afirmar, por ejemplo en la ecuación (28), que el coeficiente de 0,50799 para el logaritmo natural del tiempo, significa que por cada unidad de cambio porcentual en el tiempo, la pérdida de masa se mueve en 0,50799 por ciento, siempre y cuando se dejen fijas las demás variables. Por otro lado, el aporte de los cloruros depende de la cantidad de óxidos de azufre, ya que por unidad de cambio en el porcentaje de los cloruros, la variable dependiente se incrementa en $\hat{\beta}_{3}+\hat{\beta}_{5} \mathrm{SO}_{2}$, siendo $\hat{\beta}_{3}$ el estimador del coeficiente de regresión parcial de los cloruros y $\hat{\beta}_{5}$ el de la interacción entre los cloruros y los óxidos de azufre. Análogamente, para los óxidos de azufre, el cambio en la pérdida de masa por unidad de cambio porcentual en los óxidos de azufre, es $\hat{\beta}_{4}+\hat{\beta}_{5} \mathrm{Cl}$, donde $\hat{\beta}_{4}$ es el coeficiente correspondiente a los óxidos de azufre.

De todas las ecuaciones se infiere que aunque los coeficientes para la misma variable son bastante cercanos, unos presentan significancia y otros no; esto se debe a las diferencias entre las varianzas de los estimadores de los coeficientes respectivos.

Se debe recordar que algunas variables no consideradas en el estudio, como son las características de los ciclos de humectación y secado, la composición y tamaño de los aerosoles marinos y del material particulado, en general, la acidez de las aguas pluviales, la temperatura, la frecuencia e intensidad de éstas, etc., podrían tener efectos significativos en algunas estaciones. Su presencia en el modelo quizá mejoraría el coeficiente de deter- minación, $R^{2}$. Sin embargo, el bajo valor de la interceptación en cuatro de las otras ecuaciones (las tres de Colombia y Holguín 3), ratifica el gran significado de las variables finalmente consideradas.

Algo que queda claramente ratificado es lo relacionado con el papel de la humedad relativa en esta comparación. Las condiciones en todas las estaciones son tales que sus humedades relativas promedios superan el $80 \%$ y su fluctuación difícilmente superará el $15 \%$, tal como se encontró para las estaciones del proyecto MICAT en la zona ${ }^{[9]}$. Por otro lado, la significativa concentración de contaminantes, principalmente cloruros, minimiza el efecto de las diferencias que se tengan en las humedades relativas y el que puedan ejercer sobre los tiempos de humectación.

En Holguín 1 el tiempo influye más que en el resto de estaciones. Mamonal está cerca y la de menos influjo del tiempo es Isla Naval.

Los coeficientes de concentración de los óxidos de azufre aportan más que los de los cloruros.

La interacción es el factor de menos peso, pero tiene presencia positiva y aporte significativo (dos de las ecuaciones muestran diferencia significativa). El modelo mejora cuando se incluye la interacción.

Holguín 1, de carácter rural marino, presenta diferencias significativas en el tiempo, cloruros e intercepto. La diferencia con la estación base denota la influencia que tiene la distancia a la orilla del mar, entre las estaciones ${ }^{[37]}$. En la interceptación parece notarse, como en Viriato, el efecto de salpicadura.

Holguín 2, de carácter marino rural, no presenta diferencia significativa con respecto a Holguín 1 y Holguín 3, aunque Holguín 1 podría tener efectos de salpicadura. Las diferencias significativas nulas entre Holguín 2 y Holguín 3 permiten concluir, dada su cercanía geográfica, que estas estaciones son equivalentes, o dicho en otras palabras, que una de las dos podría eliminarse.

En Viriato hay salpicadura y su efecto parece ser mayor, pues tiene la mayor interceptación, mientras que el coeficiente de concentración de cloruros depositados por gravedad es bajo; esto sugiere que el efecto de los cloruros depositados por salpicadura es mayor que el de concentración de cloruros depositados por gravedad.

Viriato, de carácter urbano marino, a $10 \mathrm{~m}$ de la costa noroccidental cubana, presenta diferencias significativas en la interceptación y en el tiempo, no en los sulfatos, lo cual puede deberse al salpicado que se presenta y que minimizaría el efecto de otros contaminantes. 
Isla Naval es de carácter marino rural, aunque hay tránsito de embarcaciones a motor. Sólo tiene significado en la interceptación con respecto a la estación base, lo cual es lógico pues ambas tienen en lo esencial el mismo ambiente.

En suma, Isla Naval es la menos agresiva para todo, presenta diferencia significativa con la intercepción aunque es negativa.

Base Naval es de carácter marino urbano, con influencia de tránsito portuario y terrestre. Tiene diferencia significativa en interceptación, tiempo e interacción de contaminantes. El grado de contaminación podría explicar la diferencia.

La interceptación más baja es la de Mamonal; pero los coeficientes de las variables son altos y todos ellos presentan diferencias significativas con respecto a la estación base. Según esto, las variables consideradas explican mejor la variabilidad en pérdida de masa que en las otras estaciones.

El carácter de Mamonal es marino industrial, a $200 \mathrm{~m}$ de la orilla y se diferencia de la estación base (marino rural) en todos los coeficientes excepto en la interceptación.

Al buscar posibles agrupaciones, se encuentra que las estaciones de Isla Naval, Holguín 2 y Holguín 3, por condiciones climáticas y por respuesta al modelo, constituyen un conjunto de agresividad semejante. Lo mismo se puede afirmar de Base $\mathrm{Na}$ val y Viriato. Adicionalmente, Mamonal y Holguín 1 se pueden asociar, justificándolo en el hecho de que la primera está altamente afectada por la contaminación industrial, y la segunda siente el efecto de salpicadura.

\section{CONCLUSIONES}

- Se ha llegado a un modelo matemático que se ajusta de manera satisfactoria a las situaciones experimentadas por un material que se encuentra expuesto a la atmósfera, validando la causalidad de las tres variables consideradas sobre la corrosión del acero al carbono.

- El manejo dado a los datos permitió discernir el mayor o menor peso de cada una de las variables tenidas en cuenta, en la corrosividad de las diferentes estaciones, incluido el aporte con signo positivo de la interacción entre los contaminantes atmosféricos considerados.

- Como resultado de lo hallado, se puede afirmar que la serie de estaciones ubicadas en la región de Holguín, se podrían optimizar eliminando una de ellas, tal vez Holguín 2, con el consiguiente impacto, principalmente económico, que ello conllevaría. Lo anterior puede hacerse extensivo a otras situaciones, de manera que los sitios escogidos tengan características realmente diferentes, lo cual se puede decidir a partir de un corto muestreo.

- Es evidente que en el trabajo realizado no se tuvieron en cuenta muchas de las variables que influyen en la corrosión atmosférica, algunas de las cuales podrían enriquecer el modelo. Adicionalmente, hay que reconocer la existencia de otros métodos estadísticos, incluida la regresión robusta, que también podrían conducir a resultados interesantes.

- Se clasificaron y agruparon las estaciones a partir de un método estadístico que resultó coherente con las características climáticas exhibidas.

- A partir de las ecuaciones determinadas se cuenta con herramientas que permiten hacer predicciones sobre el comportamiento del acero al carbono en las estaciones estudiadas y en sitios similares de la región caribeña.

- Se ratifica la idea de que uno de los principales soportes del avance y perfeccionamiento de los conocimientos futuros en torno a los fenómenos de la corrosión atmosférica, serán las cada vez más potentes herramientas estadísticas de análisis y modelamiento.

\section{Agradecimientos}

Este proyecto se realizó en el marco de la Tercera Comisión Mixta de Cooperación Económica, Científico-Técnica, Cultural y Educativa Cubana - Colombiana y contó con el apoyo financiero del Centro Nacional de Investigaciones Científicas (Cuba) y la Universidad de Antioquía (Colombia). Se expresan los agradecimiento al profesor Luis Gabriel Agudelo y a la señorita Paola Niño Restrepo, por su colaboración en la discusión y organización de la información.

\section{REFERENCIAS}

[1] C. Arroyave, A. Valencia, F. Herrera, C. Marín, J. Delgado, J. Cuervo, J. Wilches, O. Aguirre, F. Lindado, M. Parra, G. Vila, R. Gutiérrez, C. Ramírez y J. Reyes J. Proc. 10th Asia Pacific Corrosion Control Conference, Bali, 1997 pp. 405-410.

[2] S. Feliu y M. Morcillo, Corrosión y Protección de los Metales en la Atmósfera, Edic. Bellaterra, Barcelona, 1982, $246 \mathrm{pp}$. 
[3] B.M. Rosales Anales 3er Encuentro Iberoamericano de Corrosión y Protección, Río de Janeiro, Brasil, 1989, pp. 371-381.

[4] Y.N. Mikhailovski y P.V. Strekalov, Atmospheric Corrosion. Ailor, W.H. (ed.), John Wiley and Sons, New York, EE. UU., 1982, pp. 923-942.

[5] F. Corvo, N. Betancourt, L. Muleshkova, C. Haces, Y. LeÓn, J. Pérez, E. Bricuyet y O. RodríQuezAnales 3er Encuentro Iberoamericano de Corrosión y Protección, Río de Janeiro, Brasil, 1989, pp. 405-414.

[6] M. Morcillo y S. Feliu (Eds.). Mapa de España de Corrosividad Atmosférica, CYTED, Madrid, España, 1993, 431 pp.

[7] B.M. Rosales, Mapas de Corrosividad Atmosférica de Argentina, CITEFA, Buenos Aires, Argentina, 1997, 366 pp.

[8] M.E.M. Almeida y M.G.S. Ferreira (Eds.)Corrosão Atmosférica. Mapas de Portugal, INETI, Lisboa, Portugal, 1998, 438 pp.

[9] M. Morcillo, E. Almeida, B. Rosales, J. Uruchurtu y M. MARrocos (Eds.). Corrosión y Protección de Metales en las Atmósferas de Iberoamérica. Parte I, CYTED, Madrid, España, 1999, 816 pp.

[10] L. MARiacA, J. GenesCÁ, J. Uruchurtu, y L.S. HeRnándeZ (Eds.)Corrosividad Atmosférica (MICAT-México)", Plaza y Valdés, México, D.F., Méjico, 1999, 209 pp.

[11] G.A. King, J. Kapetas, y Bates- D. Brownsword, Proc. CORROSION'94, NACE, Houston, EE. UU., 1994.

[12] G.A. KING, Mat. Perform. 34 (1995) 6-9.

[13] F.H. Haynie, y J.B. Upham, Mat. Prot. Perform. 10, 11 (1971) 18-21.

[14] M. Pourbaix, Atmospheric Corrosion, Ailor, W.H. (Ed.). , J. Wiley and Sons, New York, EE. UU., 1982, pp. 107-121.

[15] R.A. Legault, y A.G. Preban, Corrosion 31, 4 (1975) 117-122.

[16] L. Espada, P. Merino, X.R. Novoa, X.R. y A. Sánchez Rev. Iberoam. Corros. Protec. XIX 5 (1988) 285-289.

[17] F.H. Haynie y J.B. Upham. Corrosion in Natural Environments, ASTM STP 558, American Society for Testing and Materials, 1974, pp. 33-51.
[18] S. Feliu, M. Morcillo y S. Feliu Jr., Corros. Sci. 34 (1993) 403.

[19] S. Feliu, M. Morcillo y S. Feliu JR., Corros. Sci. 34 (1993) 415-422.

[20] M. Stratmann, Corros. Sci. 27 (1987) 869-872.

[21] M. Stratmann et al., Corros. Sci. 27 (1987) 905.

[22] M. Stratmann y H. Streckel, Corros. Sci. 30 (1990) 697-714.

[23] M. Stratmann, Ber. Bunsenges. Phys. Chem. 94 (1990) 626-639.

[24] ISO 9225. Corrosion of Metals and Alloys - Corrosivity of Atmospheres - Measurement of Pollution. International Standards Organisation, Geneva, Suiza, 1992.

[25] I.S. Cole, R. Holgate, P. KaO, y W. Ganther, Corros. Sci. 37 (1995) 455-465.

[26] J. Tiblad, A.A. Mikhailo, y V. Kucera, Proc. $14^{\text {th }}$ International Corrosion Congress, Cape Town, 1999.

[27] I.S Cole, W.D. Ganther y P. Norberg, Proc. $14^{\text {th }}$ International Corrosion Congress, Cape Town, 1999.

[28] L. Veleva y L. Maldonado,. Br. Corros. J. 33 (1998) 53.57.

[29] O.T. DE Rincón et al. Mater Perform. 37, 12 (1998) 48-53.

[30] A.R Mendoza y F. Corvo, Corros. Sci. 41 (1999) 75-86.

[31] L. Maldonado y L. Veleva, Werkst. und Korros. 50 (1999) 261-266

[32] D.N. GujARATI, Econometría, $3^{\text {a }}$ ed., Mc Graw-Hill, Santa Fe de Bogotá, Colombia, 1997, 624 pp.

[33] G.G. JudGE et al., Introduction to the Theory and Practice of Econometrics, John Wiley \& Sons, New York EE. UU., 1988, pp. 443-446.

[34] W.H. Green Análisis Econométrico, $3^{\mathfrak{a}}$ ed., Prentice-Hall Iberam., Madrid, 1999, p. 30.

[35] N.R. Draper, y H. Smith, Applied Regression Analysis, $3^{\text {rd }}$ ed., John Wiley \& Sons, Inc., New York, EE. UU., 1998, pp. 387-400.

[36] Shazam User's Reference Manual. Version 7.0, McGrawHill, Quebec, 1993, 483 pp.

[37] M. Morcillo, B. Chico, L. Mariaca. y E. Otero, Corros. Sci. 42 (2000) 91-104. 